\title{
NUEVA GUINEA ANTE LA AUTONOMÍA REGIONAL, UN DESAFÍO PENDIENTE
}

\author{
Claribel del Rosario Castillo Úbeda ${ }^{[1]}$ \\ Mibsam Aragón Gutiérrez ${ }^{[2]}$ \\ Luis Serra Vásquez ${ }^{[3]}$
}

\section{Resumen}

La Autonomía Regional de la Costa Caribe de Nicaragua es una temática abordada desde el enfoque de las representaciones sociales que permite conocer de que manera la población de Nueva Guinea, percibe el régimen autonómico; asimismo la identificación de posibles estrategias de definición política y administrativa de este territorio mestizo, que según la Ley 137 es parte de la Región Autónoma Atlántico Sur -RAAS-, no obstante desde el Estatuto de de las Regiones de la Costa Atlántica de Nicaragua y su Reglamento en el artículo 42, no hubo claridad en su jurisdicción.

El diseño de investigación tuvo un enfoque cualitativo, de corte transversal, cuyos instrumentos de recopilación de información fueron entrevistas individuales y grupales, con representatividad de todos los sectores sociales y políticos.

Se Identificaron diferentes apreciaciones relativas a la conceptualización y sentido de inclusión al régimen autonómico, que puede resumirse en dos posiciones. Una quienes han visto en la autonomía una oportunidad para que el municipio alcance mayores niveles de desarrollo si se integra a la región con los derechos establecidos en la Ley No. 28, y otra, quienes han pensado en una salida que implica la conformación de una nueva Región Autónoma. También se aportan elementos a las autoridades y organizaciones de la Sociedad Civil sobre el sentir de la población respecto a esta situación

Los hallazgos sugieren que la población necesita mayor divulgación de la Ley para el conocimiento de sus contenidos, y a partir de ello realizar una consulta amplia a sus habitantes antes de definir el futuro de este municipio. También se ha demandado urgentemente una respuesta clara a la indefinición política - administrativa.

Palabras claves: Representaciones sociales, régimen autonómico, mestizo, población, municipio.

\footnotetext{
[1] Licda. en Ciencias de la Educación con mención Español, MSc. con mención en Investigación e Intervención Social. Vice-rectora URACCAN Recinto Nueva Guinea.

[2] Licda. en Administración Agropecuaria, MSc. con mención en Investigación e Intervención Social. Secretaria Académica URACCAN Recinto Nueva Guinea.

[3] Phd. y tutor de la tesis.
} 


\section{Introducción}

La Autonomía Regional de la Costa Caribe de Nicaragua, es un tema objeto de investigación en el ámbito regional, nacional e internacional. Hasta hace pocos años, Nueva Guinea no fue de interés en la realización de estudios sobre la autonomía y otros temas conexos con la RAAS. Es en los últimos años que el programa para las Naciones Unidas (PNUD), en la realización del primer Informe de Desarrollo Humano de la Costa Caribe Nicaragüense (2005), incluye a estos cuatro municipios como parte de la RAAS: Muelle de los Bueyes, El Ayote, Rama y Nueva Guinea.

La Autonomía Regional es un tema ilustrado por diversos autores, debido a las particularidades del proceso, relevancia, aportes e influencia hacia los pueblos indígenas, afrodescendientes y mestizos de la región. En la revisión bibliográfica, existen profusos estudios en referencia a esta temática enfocados en la reivindicación histórica y la perspectiva de los Derechos de los Pueblos Indígenas y Comunidades Étnicas; asimismo, las dimensiones de la autonomía, establecidas en el reglamento. A su vez encontramos dos investigaciones cuantitativas, referidas al conocimiento de la Ley No. 28 y de los Gobiernos Regionales. No obstante, desde la concepción de municipio mestizo se carece de estudios por ser una zona donde la pertenencia o no a la Región Autónoma ha estado en incertidumbre y a merced de políticas estatales de corto plazo que no han dado respuestas concretas a la ciudadanía.

El objetivo de este artículo es el análisis de la problemática de la jurisdicción política -administrativa del municipio, en relación al ejercicio de los derechos autonómicos.

El referente inmediato del marco teórico corresponde a las representaciones sociales de Serge Moscovici, puesto que la autonomía regional ha sido analizada desde las percepciones que los habitantes de Nueva Guinea tienen sobre su realidad a partir del conocimiento social y las relaciones experimentadas y construidas a través de los años de vivir en este territorio.

Se formularon las siguientes preguntas guías para orientar el estudio ¿Cuál es la percepción de los habitantes de Nueva Guinea sobre la Ley de Autonomía Regional? ¿Cuáles son las creencias que tienen en relación a la Autonomía Regional? ¿Cómo sienten el ejercicio de los derechos autonómicos? ¿Cuáles son las limitaciones actuales que inciden para que la ciudadanía ejerza esos derechos? ¿Cuáles son los desafíos que presenta la plena incorporación del Municipio de Nueva Guinea a la RAAS? ¿Qué propuestas hace la población para resolver el problema de indefinición política - administrativa de Nueva Guinea?

Este estudio fue realizado en el año 2007, como tesis de graduación para optar al título de Máster en Ciencias Sociales de la Universidad Centroamericana de Nicaragua 
-UCA-. Una de sus principales limitantes fue restringirse al área urbana por razones de tiempo y económicas.

\section{Revisión de literatura}

\section{El Estado y la Autonomía Regional}

La Constitución Política de 1987, reconoce el carácter multiétnico del pueblo nicaragüense, artículo 8, en real comprensión a la diversidad étnica de la nación fundamentalmente a la existente en la Costa Atlántica. Es después del conflicto suscitado en la región (conflicto militar 1981-1985) que se genera el reconocimiento pleno por parte del Estado nicaragüense de los derechos consignados en el Estatuto de Autonomía (septiembre 1987) donde la Carta Magna incorpora como nuevo elemento el Art. 8 y el capítulo VI referido exclusivamente a los derechos de las comunidades de la Costa Atlántica.

El régimen autonómico busca construir una convivencia pacífica, entendimiento y generación de confianza mutua entre el Estado y la sociedad costeña, demanda que surgió precisamente del seno del pueblo caribeño.

Hecho referido por González (2005, p 2.):

El Estado de Nicaragua reconoce que los pueblos de la Costa han sido tratados como ciudadanos de segunda y que los derechos de ciudadanía deben ser extendidos a esa población, en pie de igualdad con los derechos del resto de los nicaragüenses. Hay también un reconocimiento a la naturaleza multiétnica de la sociedad nicaragüense y a la necesidad de que el Estado se transforme para dar cabida a esa diversidad.

El nuevo concepto de Estado derivado de la Autonomía, mencionado por Acosta (2004) a partir del análisis jurídico es planteado de la siguiente forma:

La Ley No. 28, establece la descentralización por territorio modificando la organización territorial del Estado. La creación de las dos Regiones Autónomas implica un otorgamiento de personería jurídica de derecho público que les da la capacidad de ejercer derechos, contraer obligaciones y ejercer jurisdicción como ente público territorial; con la potestad de crear actos legislativos regionales (resoluciones u ordenanzas) para ser aplicadas dentro del territorio de las Regiones Autónomas en la materia que compete a las mismas; y tener una administración pública propia. Todo lo anterior es novedoso para el ordenamiento jurídico nicaragüense que, como república unitaria, tradicionalmente había dividido el territorio nacional únicamente entre 
Departamentos y Municipios, sin que los Departamentos sean aún entes jurídicos territoriales. Ejerciendo una administración centralizada (p. 52).

También se establece en la Ley No. 28: "una concepción de dirección colegiada, plasmada en la ley a través de los Consejos Regionales como autoridades de más relevancia conjuntamente con los Coordinadores Regionales." (Ibíd., p.54) Rango que les da el hecho de ser autoridades electas en un proceso electoral celebrado a nivel regional, pero con las mismas reglas de las nacionales.

Según critica la Dra. Cunningham, se debe a la falta de voluntad política del gobierno central para apoyar las iniciativas realizadas por la ciudadanía costeña.

Otro señalamiento lo hace López y Rivas (1993, p.2) "El modelo de Estado nación en América Latina se basó en la exclusión y el monoculturalismo en todas las formas de organización del Estado".

\section{2 . Autonomía, multiculturalismo e interculturalidad}

\section{Ley de Autonomía}

La Autonomía Regional en la Costa Caribe es vista desde diferentes perspectivas y visiones. Para algunos es una reivindicación histórica exclusiva de los pueblos indígenas. Según refiere Díaz Polanco (1997, p.7) "Es el derecho de los pueblos indígenas y comunidades étnicas de elegir a sus autoridades e instrumentar efectivamente su propio modelo de desarrollo de acuerdo a su cosmovisión, cultura, tradiciones y necesidades"

Según se establece en el reglamento de la Ley No. 28, se define el régimen de autonomía como:

El sistema o forma de gobierno, jurídico, político, administrativo, económico y financieramente descentralizado que dentro de la unidad del Estado nicaragüense, establece las atribuciones propias de las Regiones Autónomas de la Costa Atlántica de Nicaragua, de sus órganos de administración, los derechos y deberes que corresponden a sus habitantes para el ejercicio efectivo de los derechos históricos de los pueblos indígenas y comunidades étnicas de la Costa Atlántica de Nicaragua, consignadas en la Constitución Política de la República de Nicaragua, la Ley No. 28 y demás leyes de la República (p. 2).

Este modelo beneficia a una población pluricultural incluyendo los mestizos que han estado en este territorio desde antes de la anexión al Estado Nacional, como se 
menciona en el Informe de Derechos Humanos de la Costa Caribe Nicaragüense (IDHCCN, 2005).

Para fines de este estudio consideramos necesario revisar las definiciones establecidas en El Reglamento a la Ley No. 28 (Ibíd. p.37, 38).

Comunidades de la Costa Atlántica o Caribe de Nicaragua: Se entiende como las entidades jurídicas, sociales, políticas, constituidas por miskitos, mayangnas o sumos, ramas, creoles, garífunas, y mestizos que habitan en las Regiones Autónomas con jurisdicción para administrar sus asuntos bajo sus propias formas de organización conforme a sus tradiciones y culturas, reconocidas en la Constitución Política y la Ley No. 28. También establece una definición propia de comunidad étnica y comunidad indígena tales como:

Comunidad étnica: "Es el conjunto de familias de ascendencia ameri-india y africana que comparten una misma conciencia étnica fácilmente identificable por su cultura, valores y tradiciones de convivencia armónica con la naturaleza, vinculados a sus raíces culturales y formas de tendencias y uso comunal de la tierra".

Comunidad indígena: "Es el conjunto de familias de ascendencia ameri-india que comparten sentimientos de identificación, vinculados a su pasado aborigen y que mantienen rasgos y valores propios de su cultura tradicional, así como formas de tendencias y uso comunal y de organización social propias". En este reglamento se establecen diferencias en cuanto a los conceptos, puesto que garífunas, creoles (de origen afrodescendientes) y mestizos, los definen como comunidad étnica.

Sin embargo, en los conceptos y definiciones básicas establecidos en el VIII censo de población y IV de vivienda establecen un concepto único para pueblo indígena y comunidad étnica, definiéndolo como "el conjunto de personas que mantienen una continuidad histórica con sus ancestros y que aún comparten tradiciones y sus propios valores culturales" (INIDE, 2005: 99).

Los datos demográficos indican que el $76 \%$ de la población de las Regiones Autónomas es mestizo e hispano parlante. Ese fenómeno biodemográfico, social, político y cultural presenta profundos retos al modelo actual de régimen de autonomía y a los principios de reconocimiento a los derechos históricos de pueblos indígenas y comunidades étnicas, representación étnica incluyente y acciones afirmativas para garantizar igualdad en el ejercicio de derechos y deberes en la institucionalidad autonómica. En la RAAS la población mestiza es aún mayor, tan es así que el 90\% de su población es mestiza (IDHCC, 2005: p.59). 


\subsubsection{Multiculturalidad e interculturalidad}

La multiculturalidad se refiere a la coexistencia de dos o más culturas en un territorio y período de tiempo determinado, cada una de ellas conservando todos o algunos de sus rasgos distintivos en dependencia de su estatus dentro de la jerarquía etno lingüística (Del Cid, 2002).

El multiculturalismo impulsa "acciones afirmativas" como medidas promovidas por las sociedades dominantes con el propósito de amortiguar los impactos, de lo que perciben como efectos negativos de estas sociedades marginales, sobre las sociedades de poder establecidas. Constituyen además medidas transitorias que permiten ir creando las condiciones para la asimilación de estas culturas hacia la sociedad dominante. Ello lleva implícito entre otras cosas la pérdida total o el debilitamiento de la identidad y de los valores culturales en estas sociedades marginales.

La Interculturalidad se entiende como un proceso de negociación social que, a partir de una realidad fuertemente marcada por el conflicto y las relaciones asimétricas de poder, busca construir relaciones dialógicas justas entre los actores sociales pertenecientes a universos culturales diferentes, sobre la base del reconocimiento de la diversidad (Moya, 2003: p. 106).

\section{Materiales y métodos}

Se trata de una investigación cualitativa, exploratoria, que aborda desde las representaciones sociales las percepciones acerca de la Autonomía Regional por parte de la ciudadanía de Nueva Guinea. Es un estudio de corte transversal porque se realizó durante el año 2007, y propositiva por brindar algunas recomendaciones que contribuirán a superar la problemática identificada.

Para la recolección de la información se trabajó con fuentes primarias, proporcionada por los grupos focales y entrevistas, y secundarias a través de la revisión bibliográfica e internet.

Para la selección de los grupos focales se tomaron en cuenta algunas características como: estudiantes de ambos sexos mayores de 16 años, de los primeros años de todas las carreras y habitantes del área urbana de Nueva Guinea; docentes permanentes y horarios de ambos sexos de la universidad URACCAN Nueva Guinea, con menos de dos años de trabajar para la institución. El grupo focal en la sociedad civil, se conformó en base a los criterios de representatividad institucional, liderazgo político y social, relación de dependencia institucional.

El análisis de resultados se realizó mediante una matriz agrupando los resultados por categorías. La realización del análisis e interpretación de la información se hizo 
aplicando el método de teorización que nos permitió percibir, contrastar e interpretar las categorías; establecer nexos, y relaciones entre la información brindada y la teoría.

\section{Resultados y discusión}

\section{Conocimiento sobre la Ley de Autonomía}

El estudio refleja que hay un cierto grado de conocimiento acerca del concepto autonómico. La mayoría de los entrevistados expresaron tener información de manera general sobre la Autonomía Regional. Es decir han oído hablar de la autonomía, pero no están bien claros sus significados y alcances de la ley.

Los estudiantes integrantes del grupo focal expresaron tener conocimientos acerca de la Ley de Autonomía, pero hicieron énfasis en el aislamiento, falta de oportunidades y de participación a los cuales tienen derecho; pero, por su condición de habitantes de este municipio no aplica para ellos. "Los de Nueva Guinea estamos aislados porque no nos incluyen en el Gobierno Regional, no tenemos derecho a votar" También reconocieron que aunque territorialmente el municipio pertenece a la Región Sur, hay poca o ninguna participación "El objetivo es que todos participemos, que valoren nuestra idiosincrasia. La Ley de Autonomía nos abarca a todos pero no se aplica" (Grupo focal estudiantes).

\section{Conceptos sobre Autonomía Regional}

Tanto en los grupos focales como en las entrevistas individuales los conceptos expresados denotaron el dominio y conocimiento acerca del significado de la autonomía. Las expresiones al respecto así lo indican.

Reconocimiento de los derechos sociales, educativos y económicos a la ciudadanía de esta región. Así como el reconocimiento a su cultura. (Entrevista a delegado institucional).

Son las normas y leyes constitucionales que le permiten la expresión a la ciudadanía nicaragüense que estaban segregados y esta ley da las facultades para que les permitan el desarrollo humano. La autonomía se ve como el empoderamiento de los habitantes del Caribe nicaragüense. (Entrevista a delegada Municipal, institución gubernamental).

A través de estas expresiones se refleja un manejo del concepto autonómico, no sólo desde el enfoque de reconocimiento a los derechos de los pueblos que habitan la región, sino también a la oportunidad de disponer de leyes que permitan alcanzar sus propios niveles de desarrollo, relacionado también con mejorar las condiciones 
de vida. Estos conceptos se relacionan y coinciden con lo descrito en el Informe de Desarrollo Humano, en el cual se afirma que la Autonomía Regional no es un régimen que fomenta el separatismo ni desconoce la institucionalidad nacional. Esta afirmación es ratificada por los entrevistados cuando afirman, "el régimen autonómico fomenta sus propias leyes, pero dentro de un mismo Estado nación” (Entrevista miembro organización comunitaria).

Las opiniones sobre las definiciones de autonomía, se asocian a factores diversos como: nivel educativo, nivel de relaciones y de conocimiento obtenido por las tareas o funciones ejercidas en calidad de representantes de instituciones gubernamentales y no gubernamentales o partidos políticos.

Algunas personas entrevistadas dejaron ver que la autonomía es para los otros, "Es una forma de gobernar sus propios bienes", "nació como una necesidad de los pueblos indígenas de la costa caribe", "Es la normativa que regula la capacidad propia de la región para gobernarse por sí misma". Solamente dos personas de las entrevistadas se refirieron al concepto, desde el sentido de la inclusión, es decir no se referían a la autonomía como una ley única y exclusivamente para los otros, sino que hicieron alusión a ellos mismos como parte integrante de la autonomía. "La autonomìa es conservar nuestras propias costumbres", "es cuando somos capaces de decidir, de administrar los recursos, los bienes y todo lo que tenemos en un determinado lugar" en estas expresiones vemos las palabras "nuestras" $y$ "somos" como signos importantes de identificación e inclusión.

\section{Sentido de pertenencia}

Las características particulares de poblamiento de los habitantes de Nueva Guinea, cuyos orígenes están basados en los procesos de colonización espontánea o planificada, impulsada por el Instituto Agrario Nacional en los años sesenta, hacen de esta población una comunidad en proceso de desarrollo y formación de su identidad.

En este contexto y para fines de este estudio indagamos a través de la interrogante siguiente: ¿Cómo ciudadano de Nueva Guinea se siente usted parte de la RAAS? Las respuestas fueron diversas. Miembros del grupo focal conformado por integrantes de la Sociedad Civil organizada respondieron categóricamente no sentirse parte de la RAAS por diferentes factores: se consideran una población con características culturales diferentes. Otro factor es la ubicación geográfica de Nueva Guinea cuya distancia y costos de accesibilidad a Bluefields como sede de la Región es escasa. Y finalmente señalan la falta de comunicación y relaciones entre las instituciones gubernamentales y no gubernamentales del municipio con sus homólogas en la sede regional.

En los grupos focales nos encontramos con dos posiciones: por un lado los jóvenes y el personal docente manifestaron sentirse parte de la RAAS, aunque no se les toma 


\section{AUTONOMÍA}

en cuenta y por otro, los líderes políticos, un sector la Sociedad Civil, y directores de instituciones en su mayoría, son quienes señalan no sentirse parte de la RAAS, debido a: falta de atención de los gobiernos regionales y relaciones de coordinación institucional realizadas tradicionalmente con Chontales.

Los estudiantes a diferencia de las demás personas entrevistadas mayores de edad y procedentes originalmente de otras partes del país, sí expresan sentimientos de arraigo al territorio donde viven, y ponen énfasis en sus palabras cuando afirman "sí, porque es donde vivimos" "somos parte de ella" (la región). Es decir, el municipio es parte porque territorialmente está contemplado en la Ley No. 59 -Ley de división política administrativa-. Pero, señalan falta de atención de las autoridades regionales para esta población.

Un aspecto reiterativo expresado por la mayoría de los informantes fue la reafirmación de su identidad como mestizos, "somos diferentes" son las expresiones frecuentes, en comparación con los otros pueblos que habitan la región, debido a las diferencias culturales, étnicas y lingüísticas muy particulares que tienen los pueblos del litoral. La misma falta de comunicación y relación entre este municipio y los municipios costeros son también aspectos mencionados por los entrevistados que hacen profundizar las diferencias y el sentimiento de no pertenencia a la región.

Son los jóvenes quienes identificaron su pertenencia al territorio. Esto puede obedecer a varios factores. En primer lugar, son una generación nacida en el municipio, no así sus padres quienes en su mayoría nacieron en otros departamentos del país y aunque muchos llegaron aún en su niñez, no reconocen su pertenencia territorial a la RAAS.

También, cuentan con un nivel educativo mayor en algunos casos a los políticos y ciudadanía en general, puesto que son estudiantes universitarios y quizá estén influidos por las políticas de integración que promueve la URACCAN y por las relaciones con los demás Recintos Universitarios. Un último factor sería la facilidad de acceso a los medios informativos, documentos y leyes en los cuales se expresa la situación de pertenencia legal del municipio a la RAAS.

El sentido de pertenencia o no a la RAAS no es una posición exclusiva de los habitantes del municipio de Nueva Guinea, El doctor Jorge Grünberg lo plantea cuando expresa:

Una buena parte de la población mestiza de la frontera agrícola no percibe encontrarse en la Costa Caribe. Eso está claro en el caso de Nueva Guinea y hasta El Rama. Muchos chontaleños, boaqueños, etc. conocen "La Guinea" pues ahí van y compran ganado, queso, etc., pero nunca van a decir que fueron a la Costa Caribe. 


\section{Identificación con la Ley de Autonomía}

La totalidad de las personas participantes en el estudio hacen énfasis de no sentirse incluidos y sus comentarios redundan que como ciudadanas y ciudadanos y como municipio en sí, no han sido tomados en cuenta.

También hacen alusión a que esta ley excluye a los mestizos y está diseñada sólo para los otros grupos étnicos existentes en la Costa Caribe. En el Informe de Desarrollo Humano se habla de dos tipos de mestizos que habitan la Costa Caribe nicaragüense: los mestizos costeños, son aquellos que han vivido por generaciones y se sienten identificados con las costumbres y culturas de los pueblos que la habitan y los mestizos llegados en los últimos años empujados por la frontera agrícola. Según expresiones brindadas por algunos entrevistados se sienten diferentes al resto de los habitantes y se perciben así mismos como personas con costumbres y cultura distinta a la del resto de la región caribeña. Por lo tanto, no se identifican como mestizos costeños. “...No me siento identificado, no participamos dentro de ese proceso autónomo, no tenemos representantes en el Gobierno Regional, existe la ley pero nosotros somos excluidos" (Entrevista a líder político).

\section{Limitaciones en la aplicación de la Ley de Autonomía Regional}

Se logró identificar varias limitaciones y en primera instancia está el factor voluntad política: existe una percepción generalizada de los participantes en el estudio sobre la inminente falta de voluntad y juego de intereses en la resolución del problema de definición política administrativa de esta comunidad y aducen entre otras cosas que:

En los períodos de campaña el tema de la indefinición de Nueva Guinea se vuelve importante. Pasado este tiempo, pierde relevancia para los políticos la problemática que mencionan los entrevistados. Es así como se ha convertido en un asunto de interés electoral con resultados no tangibles a la fecha para sus habitantes. La siguiente expresión así lo categoriza:

No se ha puesto interés en el tema. Los políticos se aprovechan de esto para sus campañas, y prometen a la gente crear un nuevo departamento y/o región porque la gente quiere que este tema se resuelva, pero falta acercamiento con las entidades regionales. El Gobierno Regional y otras instancias no han hablado de la autonomía (Grupo focal, docentes de URACCAN).

Como segundo factor está el étnico-cultural: la totalidad de las personas informantes de esta investigación lo perciben como un aspecto que los hace diferentes a los de aquí -Nueva Guinea- y los de allá -Bluefields-, y sobre esto mencionan "su lengua es otra", y esto constituye una limitante en la comunicación y acercamiento entre los habitantes de uno y el otro lugar. También señalan que las "costumbres de 
los costeños", son distintas a las que practican la ciudadanía de Nueva Guinea. Esto se puede ver en la expresión siguiente: "No comparto la cultura con los costeños porque no hablo inglés ni sus otras lenguas" (entrevista a líder político).

\section{La comunicación, el acceso y costos}

Las comparaciones redundan aquí en el aspecto de que tanto cuesta en términos de tiempo, transportación y costos viajar a Chontales o a Bluefields, como las dos ciudades principales donde se concentran instituciones gubernamentales que la población demanda servicios, por ejemplo registrar propiedades. Y mencionan esto: "La accesibilidad a Bluefields es menor, porque la vida es muy cara, los costos de trasporte son altos y la atención es mala" (grupo focal de la Sociedad Civil).

\section{Ventajas de pertenecer a un territorio autonómico}

Se identificaron ventajas en el aspecto político, económico, social y cultural. En el aspecto político muchos señalan que a partir de la inclusión de Nueva Guinea en la ley de autonomía regional, sus ciudadanos podrán elegir y ser electos en el Consejo Regional. En el aspecto económico, señalan la inclusión en el presupuesto regional, mayor comercio y acceso de sus productos. En lo social se definiría de una vez la pertenencia al territorio y en el aspecto cultural, habría una gran riqueza dado el intercambio entre culturas y se daría paso a una verdadera interculturalidad.

\section{Desventajas}

Algunos entrevistados expresaron que habría algunas desventajas en caso de que Nueva Guinea sea tomada en cuenta como un municipio que goce de los derechos autonómicos. Estas desventajas podrían estar dadas por la distribución de los recursos presupuestarios los cuales serían limitados debido a que pasarían primero por el Gobierno Regional y luego al municipio; y con la experiencia existente hasta la fecha, donde hay poca vinculación entre los gobiernos regionales y esta comunidad no habría prioridad, ni equidad en la asignación de recursos.

Otro aspecto desventajoso según ellos es la capacidad de gestión y movilización, la cual sería más lenta por el problema del acceso vía río Escondido y señalan también el aspecto cultural porque afirman sentirse diferentes.

\section{Valoraciones y propuestas}

La mayoría de informantes en esta investigación están de acuerdo en que es urgente la solución a la situación de este municipio en cuanto a la definición de atención y pertenencia en lo político y administrativo. Las posiciones son que es competencia del Gobierno Local, Regional y Central resolver este caso. Otra opinión es que se 
conforme una comisión integrada por personalidades del Gobierno Local, Sociedad Civil y universidades y que este órgano gestione ante las instancias correspondientes (Consejo Regional, Asamblea Nacional) la definición y solución al tema en mención.

En general, las respuestas están orientadas a definir por orden de competencias las instancias responsables a ofrecer una respuesta a la problemática que enfrenta este municipio. Es decir hay un reconocimiento que para resolver la definición política-administrativa de esta comunidad se requiere de la intervención de todos los sectores.

Las propuestas son diversas y van desde el aspecto jurídico: que consiste en reformar las leyes, darlas a conocer, y que la Asamblea Nacional lo ventile y resuelva. En otro aspecto plantean la creación de nuevas estructuras (comisión) que gestione y promueva ante las diversas instancias la pronta salida a la indefinición del municipio. Y por último se hace énfasis en una mayor participación de la población, partidos políticos, universidades, gobiernos locales y regionales para hacer extensas las inquietudes ante el Poder Ejecutivo y Legislativo del país.

\section{Conclusiones}

La percepción de la ciudadanía de Nueva Guinea acerca de la Autonomía Regional, consiste en el reconocimiento de la Ley No. 28 , la cual consideran afirmativamente como un instrumento legal que reconoce a los pueblos indígenas y comunidades étnicas que habitan el Caribe nicaragüense; sin embargo, hay un sentimiento de exclusión y aislamiento en relación a los municipios del Caribe, producto de la indefinición política administrativa y la falta de voluntad de las autoridades nacionales, regionales y locales para lograr la inclusión definitiva del territorio al resto de la región.

Algunos consideran que por el hecho de pertenecer a una cultura mestiza y provenir de diversas partes del territorio nacional, les hace sentir diferentes y no ven en la Autonomía Regional un proyecto que les permita lograr sus perspectivas de progreso. Mientras hay quienes ven en el régimen autonómico una oportunidad para alcanzar cambios sustanciales en la gestión y desarrollo de este municipio.

Las diversas formas de dependencia de las instituciones presentes en el municipio de Nueva Guinea ha acentuado el sentimiento de aislamiento y frustración de su población al no tener claramente establecida la dependencia administrativa y política de las distintas instituciones estatales que laboran en este municipio. La ciudadanía en general expresa el sentirse utilizados para fines políticos (cuando se está en período electoral y se busca sumar votos para alcanzar la cuota de diputados correspondiente a la región) y económicos, cuando se desea presentar a la RAAS con una mayor cantidad de población para gestionar recursos. 
Aún cuando la autonomía regional no es un tema que se aborda en las agendas locales ni está presente en los medios de comunicación, se logró identificar que existe un conocimiento mínimo sobre su concepto y significado.

Las propuestas que surgen de esta investigación son: revisión y adecuación del marco jurídico a la Ley No. 28, específicamente lo concerniente a clarificar las condiciones para que estos territorios sean incorporados con todos los derechos que faculta la misma. La ley electoral, Ley No. 211 debe ser objeto de reforma en los artículos 148 y 142 para reordenar las circunscripciones electorales e incluir a Nueva Guinea y el resto de municipios que a la fecha no participan ni están incluidos.

También debe retomarse el decreto presidencial 33-2001 que mandata a reincorporar los municipios (El Ayote, El Rama, Muelle de los Bueyes y Nueva Guinea) que estaban fuera de la jurisdicción de la RAAS.

Finalmente se debe hacer una divulgación masiva de la ley y sus alcances, seguido de una consulta a la población para luego tomar una decisión final sobre el tema.

\section{Lista de referencias}

Acosta, M. L. (2004) Régimen legal de la de las Regiones Autónomas de la Costa Caribe Nicaragüense. Universidad de las Regiones Autónomas de la Costa Caribe de Nicaragua (URACCAN) 1a ed. Editarte, Managua.

Asamblea Nacional (1987). Estatuto de de las Regiones de la Costa Atlántica de Nicaragua (Ley No. 28) Decreto No 3584.

Asamblea Nacional de la República de Nicaragua (2003). Constitución Política de la República de Nicaragua con sus reformas, 9na. Edición, Editorial Jurídica, Managua.

Asamblea Nacional de la República de Nicaragua (1991). Ley 137, ley de reforma a la Ley de División Política Administrativa.

Asamblea Nacional de la República de Nicaragua (2003). Reglamento a la Ley 28, 9 de julio del dos mil tres.

Del Cid, V. M. (2002) Pautas para la elaboración de indicadores en interculturalidad. Documento, URACCAN.

Díaz, H. (1997). Díez años de autonomía en Nicaragua. ALAI, América Latina en Movimiento. Publicado en ALAI 258, view, 7 Pág. 
González, M. (1997) Gobiernos pluriétnicos: la constitución de regiones autónomas en Nicaragua. Edit. Plaza y Valdés \& URACCAN.

Grünberg, J. (2003). Metodología de investigación Participativa Intercultural con Enfoque de Género (Postgrado de Especialización). CEIMM, URACCAN.

INIDE (2005) VIII censo de población y IV de vivienda.

Mora, M. (2002). La teoría de las representaciones sociales de Serge Moscovici. Universidad de Guadalajara, México.

Ortega, M. (1977) El Régimen de autonomía en Nicaragua: contradicciones históricas y debates recientes. Documento

PNUD. (2005). Informe de Desarrollo Humano 2005. Las Regiones Autónomas de la Costa Caribe. ¿Nicaragua asume su diversidad. Programa de las Naciones Unidas para el desarrollo. 1 ed. Managua.

Universidad URACCAN \& BICU (Relatoría General-2004). Memoria Del IV Simposio Internacional de de las Regiones Autónomas de la Costa Caribe Nicaragüense.

\section{Revistas y periódicos}

Manzo, L. \& Westerbout, M. C. (2003) Propuesta metodológica en Educación Intercultural para Contextos Urbanos. Cuadernos Interculturales y del patrimonio. Alo 1. No. 1. Septiembre.

\section{Revista electrónica}

Moya, R. (1998). Reformas Educativas e interculturalidad en América Latina. Revista Iberoamericana de Educación No. p: 17: 105-187. www.campus.oei.org/oeivirt17. htm. 